\title{
A Simple Proof of Kwapien's Theorem
}

\author{
By \\ Yasuo YAMASAKI*
}

The purpose of the present note is to prove in a self-contained way Kwapien's theorem that gives a criterion for Hilbertizability of normed spaces. Original proof [1], [2] has required much more complicated discussions. Many authors have investigated [3] [6] relating problems, but their researches are based on mutual quotations, thus it is difficult to follow their studies for readers not so familiar in the topic. So the author believes that a self-contained proof has some meaning.

\section{§1。 KKwapien's Theorem}

Theorem If a real Banach space $E$ is of type 2 and cotype 2, then $E$ is isomorphic to a Hilbert space.

Proof. The definition of type 2 and cotype 2 is:

$$
{ }^{g} a, b>0{ }^{\mathrm{V}}{ }^{\mathrm{V}} x_{1}, x_{2}, \cdots, x_{n} \in E \text { (except } x_{1}=x_{2}=\cdots=x_{n}=0 \text { ) }
$$

$$
a \sum_{i=1}^{n}\left\|x_{i}\right\|^{2}<\int\left\|\sum_{i=1}^{n} \varepsilon_{i} x_{i}\right\|^{2} d m(\varepsilon)<b \sum_{i=1}^{n}\left\|x_{i}\right\|^{2}
$$

where $\varepsilon=\left(\varepsilon_{i}\right)_{i=1}^{n}, \varepsilon_{i}= \pm 1$ and $m$ is the product measure of $\frac{1}{2}\left(\delta_{1}+\delta_{-1}\right), \delta_{1}$ being the Dirac measure placed at 1.

In (1.1), the first inequality is the definition of cotype 2 , while the second inequality is the definition of type 2 .

First step From (1.1) we can derive

$$
a \sum_{i=1}^{n}\left\|x_{i}\right\|^{2}<\int\left\|\sum_{i=1}^{n} t_{i} x_{i}\right\|^{2} d g(t)<b \sum_{i=1}^{n}\left\|x_{i}\right\|^{2}
$$

where $t=\left(t_{i}\right)_{i=1}^{n}$ and $g$ is the product measure of one-dimensional gaussian measures with variance 1 .

$\mathbb{R}^{n}$ can be written as the product $\mathbb{R}^{n}=\mathbb{R}_{+}^{n} \times\{-1,1\}^{n}$, and the measure $g$ can be written as $g=g_{+} \times m$, where $g_{+}$is the (normalized) gaussian measure on

Received October 18, 1983.

* Research Institute for Mathematical Sciences, Kyoto University, Kyoto 606, Japan. 
$\mathbb{R}_{+}^{n}=\left\{\left(t_{i}\right) \mid t_{i} \geqq 0\right.$ for $\left.^{\forall} i\right\}$. Therefore we get

$$
\int\left\|\sum_{i=1}^{n} t_{i} x_{i}\right\|^{2} d g(t)=\iint\left\|\sum_{i=1}^{n}\left|t_{i}\right| \varepsilon_{i} x_{i}\right\|^{2} d g_{+} d m .
$$

Assuming (1.1) we have

$$
(1.3)<b \int \sum_{i=1}^{n} t_{i}^{2}\left\|x_{i}\right\|^{2} d g_{+}=b \sum_{i=1}^{n}\left\|x_{i}\right\|^{2} \int t_{i}^{2} d g_{+}=b \sum_{i=1}^{n}\left\|x_{i}\right\|^{2} .
$$

In a similar way, also we have (1.3)>a $\sum_{i=1}^{n}\left\|x_{i}\right\|^{2}$. Thus we have proved (1.2).

Second step Assume that $x_{1}, x_{2}, \cdots, x_{n}, y_{1}, y_{2}, \cdots, y_{n} \in E$ and

$$
\sum_{i=1}^{n} f\left(x_{i}\right)^{2}=\sum_{i=1}^{n} f\left(y_{i}\right)^{2} \quad \text { for }{ }^{\mathrm{v}} f \in E^{*} .
$$

Then we have

$$
\int\left\|\sum_{i=1}^{n} t_{i} x_{i}\right\|_{1}^{2} d g(t)=\int\left\|\sum_{i=1}^{n} t_{i} y_{i}\right\|^{2} d g(t) .
$$

Denote with $g_{x_{1}, x_{2}, \cdots, x_{n}}$ the measure on $E$ induced by the mapping $t=\left(t_{i}\right) \rightarrow$ $\sum_{i=1}^{n} t_{i} x_{i} \in E$ from the measure $g$. Then the characteristic function of $g_{x_{1}, x_{2}, \cdots, x_{n}}$ is

$$
\begin{aligned}
\chi_{x_{1}, x_{2}, \cdots, x_{n}}(f) & =\int_{E} \exp [i f(x)] d g_{x_{1}, x_{2}, \cdots, x_{n}}(x)=\int_{\mathbb{R}^{n}} \exp \left[i f\left(\sum_{i=1}^{n} t_{i} x_{i}\right)\right] d g(t) \\
& =\int_{\boldsymbol{R}^{n}} \exp \left[i \sum_{i=1}^{n} t_{i} f\left(x_{i}\right)\right] d g(t)=\exp \left[-\sum_{i=1}^{n} f\left(x_{i}\right)^{2} / 2\right] .
\end{aligned}
$$

Therefore under the assumption of (1.4), we have $\chi_{x_{1}, x_{2}, \cdots, x_{n}}(f)=\chi_{y_{1}, y_{2}, \cdots, y_{n}}(f)$. From the one-to-one correspondence between the characteristic function and the measure, we see that $g_{x_{1}, x_{2}, \cdots, x_{n}}=g_{y_{1}, y_{2}, \cdots, y_{n}}$. Hence

$$
\begin{aligned}
\int_{\mathbb{R}^{n}}\left\|\sum_{i=1}^{n} t_{i} x_{i}\right\|^{2} d g(t) & =\int_{E}\|x\|^{2} d g_{x_{1}, x_{2}, \cdots, x_{n}}(x)=\int_{E}\|x\|^{2} d g_{y_{1}, y_{2}, \cdots y_{n}}(x) \\
& =\int_{\mathbb{R}^{n}}\left\|\sum_{i=1}^{n} t_{i} y_{i}\right\|^{2} d g(t),
\end{aligned}
$$

namely we get (1.5).

Third step Let $C\left(S^{*}\right)$ be the Banach space of weakly continuous functions on the unit sphere $S^{*}$ of $E^{*}$. For every $x_{1}, x_{2}, \cdots, x_{n} \in E$, we shall define $\Phi_{x_{1}, x_{2}, \cdots, x_{n}} \in C\left(S^{*}\right)$ as follows:

$$
\Phi_{x_{1}, x_{2}, \cdots, x_{n}}(f)=\sum_{i=1}^{n} f\left(x_{i}\right)^{2}
$$

Combining (1.2) with the result of Second step, we see that 


$$
\Phi_{x_{1}, x_{2}, \cdots, x_{n}}=\Phi_{y_{1}, y_{2}, \cdots, y_{m}} \text { implies } a \sum_{i=1}^{n}\left\|x_{i}\right\|^{2}<b \sum_{i=1}^{m}\left\|y_{i}\right\|^{2} \text {. }
$$

Even if $n>m$, we can add $y_{m+1}=y_{m+2}=\cdots=y_{n}=0$ to prove (1.7).

Fourth step Consider a subset $A$ of $C\left(S^{*}\right)$ such that

$$
A=\left\{\Phi_{x_{1}, x_{2}, \cdots, x_{n}} \mid \sum_{i=1}^{n}\left\|x_{i}\right\|^{2}=1\right\}
$$

$A$ is convex, because $\lambda \Phi_{x_{1}, x_{2}, \cdots, x_{n}}+(1-\lambda) \Phi_{y_{1}, y_{2}, \cdots, y_{m}}=\Phi_{\sqrt{\lambda} x_{1}, \cdots, V \bar{\lambda}_{x_{n}}, \sqrt{1-\lambda} y_{1}, \cdots, V \overline{1-\lambda}} y_{m}$ and $\sum_{i=1}^{n}\left\|\sqrt{\lambda} x_{i}\right\|^{2}+\sum_{i=1}^{m}\left\|\sqrt{1-\lambda} y_{i}\right\|^{2}=\lambda \sum_{i=1}^{n}\left\|x_{i}\right\|^{2}+(1-\lambda) \sum_{i=1}^{m}\left\|y_{i}\right\|^{2}=1$.

(1.7) means $b A \cap a A=\phi$, because if $b \Phi_{x_{1}, x_{2}, \cdots, x_{n}}=a \Phi_{y_{1}, y_{2}, \cdots, y_{m}}$ with $\sum_{i=1}^{n}\left\|x_{i}\right\|^{2}=$ $\sum_{i=1}^{m}\left\|y_{i}\right\|^{2}=1$, then we get $\Phi_{\sqrt{b} x_{1}, \sqrt{b}} x_{2}, \cdots, \sqrt{b} x_{n}=\Phi_{\sqrt{a} y_{1}, \sqrt{a} y_{2}, \cdots, \sqrt{a} y_{m}}$ with $\sum_{i=1}^{n}\left\|\sqrt{b} x_{i}\right\|^{2}$ $=b$ and $\sum_{i=1}^{m}\left\|\sqrt{a} y_{i}\right\|^{2}=a$, which contradicts to (1.7).

Fifth step Since $A$ is a convex set and $b A \cap a A=\phi$, by the corollary of the separation theorem (the proof is given later) there exists a linear function $F$ which is positive on $A$ and satisfies

$$
a \sup _{\Phi \in A} F(\Phi) \leqq b \inf _{\Phi \in A} F(\Phi)
$$

Using this $F$, we shall define $(x, y)_{H}=F(f(x) f(y)) . \quad(x, y)_{H}$ is bilinear and $(x, x)_{H}$ $=F\left(\Phi_{x}\right)>0$, therefore $\|x\|_{H}=\sqrt{(x, x)_{H}}$ is a Hilbertian norm.

From (1.9) we get

$$
a \sup _{x \in S}\|x\|_{H}^{2} \leqq b \inf _{x \in S}\|x\|_{H}^{2} .
$$

So, replacing $\|\cdot\|_{H}$ by its suitable constant multiple, we can suppose that $a \leqq\|x\|_{H}^{2} \leqq b$ on $S$, or equivalently

$$
a\|x\|^{2} \leqq\|x\|_{H}^{2} \leqq b\|x\|^{2} \quad \text { for }{ }^{v} x \in E .
$$

This means that $E$ is isomorphic to the Hilbert space whose norm is $\|\cdot\|_{H}$.

(q.e.d.)

\section{§2. Separation Theorem}

Theorem Let $N$ be a real normed space and $U$ be its open unit ball. If $A$ is a convex subset of $N$ such that $A \cap U=\phi$, then there exists a linear function Fon $N$ such that

$$
\|F\|^{*} \leqq 1, \quad{ }^{\forall} x \in A \quad F(x) \geqq 1 .
$$

Proof. First step If $N$ is finite dimensional, then two disjoint compact 
convex sets $A$ and $B$ can be separated by a hyperplane.

Since $N$ is isomorphic to $\boldsymbol{R}^{n}$, we shall adopt the Euclidean metric. There exists $x_{0} \in A$ and $y_{0} \in B$ such that $d\left(x_{0}, y_{0}\right)=d(A, B)$ (=the shortest distance between $A$ and $B$ ). Let $l$ be the line connecting $x_{0}$ and $y_{0}$. Then the hyperplane $\pi$, which is orthogonal to $l$ and passes through $\left(x_{0}+y_{0}\right) / 2$, separates evidently $A$ and $B$.

Second step In general case, let $B^{*}$ be the closed unit ball of $N^{*}$. $B^{*}$ is weakly compact. For $x \in A$ and $\varepsilon>0$, we shall put

$$
K_{x, \mathrm{\varepsilon}}=\left\{F \in B^{*} \mid F(x) \geqq 1-\varepsilon\right\} .
$$

For every $x_{1}, x_{2}, \cdots, x_{n} \in A$ and $\varepsilon>0$, we have

$$
\bigcap_{k=1}^{n} K_{x_{k}, \mathrm{e}} \neq \phi,
$$

because the convex hull of $\left\{x_{1}, x_{2}, \cdots, x_{n}\right\}$ is finite dimensional and disjoint with the closed ball of radius $1-\varepsilon$, so that First step is applicable. (A linear continuous function on a subspace of $N$ can be extended on $N$ without changing its norm, according to Hahn-Banach's theorem).

Since $B^{*}$ is weakly compact, the finite intersection property implies the complete intersection property, therefore

$$
\bigcap_{\varepsilon>0} \bigcap_{x \in A} K_{x, \varepsilon} \neq \phi \text {. }
$$

Take an element $F$ from this set, then evidently we have (2.1).

Corollary Let $E$ be a real vector space, $A$ be a convex set and suppose that $A \cap a A=\phi$ for some $a>1$. Then there exists a linear function $F$ such that

$$
\sup _{x \in A} F(x) \leqq 1 \leqq a \inf _{x \in A} F(x) .
$$

Proof We can assume that $A$ spans $E$, because a linear function on a subspace of $E$ can be extended on $E$.

Since $A$ is convex, every element $z$ of $E$ can be written in the form:

$$
z=\lambda x-\mu y, \quad x, y \in A, \lambda \geqq 0, \mu \geqq 0 \text {. }
$$

Define $\|z\|$ by

$$
\|z\|=\inf \{\lambda+\mu \mid z=\lambda x-\mu y, x, y \in A, \lambda \geqq 0, \mu \geqq 0\} .
$$

Then $\|\cdot\|$ becomes a semi-norm on $E$. (It may not be a norm, but the following discussions are kept valid considering the factor space $E /\{z|| z||=0\}$ ).

Suppose that $z=a x \in a A$. If $a x=\lambda x^{\prime}-\mu y^{\prime}$ for some $x^{\prime}, y^{\prime} \in A$ and $\lambda \geqq 0$, 
$\mu \geqq 0$, we have $x^{\prime}=\frac{a}{\lambda} x+\frac{\mu}{\lambda} y^{\prime} \in \frac{a+\mu}{\lambda} A$. Since $A \cap a A=\phi$, this implies $\frac{a+\mu}{\lambda}<a$, so that $\lambda>1$. Thus $z \in a A$ implies $\|z\| \geqq 1$.

By the separation theorem just proved, there exists a linear function $F$ such that $\|F\|^{*} \leqq 1$ and ${ }^{v} z \in a A \quad F(z) \geqq 1$ or equivalently ${ }^{v} x \in A \quad a F(x) \geqq 1$. Since $x \in A$ implies $\|x\| \leqq 1$, we have evidently ${ }^{\mathrm{V}} x \in A \quad F(x) \leqq 1$. This completes the proof of (2.5).

\section{References}

[1] Kwapien, S., Isomorphic characterization of inner product spaces by orthogonal series with vector valued coefficients, Studia Math., 44 (1972), 583-595.

[2] Lindenstrauss, J. and Pelczynski, A., Absolutely summing operators in $\mathcal{L}_{p}$ spaces and their applications, Studia Math., 29 (1968), 275-326.

[3] Maurey, B., Théorèmes de factorisation pour les opérateurs linéaires à valeurs dans les espaces $L^{p}$, Astérisque $n^{\circ} \mathbb{1 1}$ Soc. Math. France (1974).

[4] Pisier, G., Grothendieck's theorem for non-commutative $C^{*}$-algebras with an appendix on Grothendieck's constant, J. Funct. Anal., 29 (1978), 397-415.

[5] Blei, R., A uniformity property for $\Lambda(2)$ sets and Grothendieck's inequality, Symposia Math., 50 (1977), 321-336.

[6] Figiel, T., Lindenstrauss, J. and Milman, V., The dimension of almost spherical sections of convex bodies, Acta Math., 139 (1977), 53-94. 
